\title{
Nota técnica
}

\section{APROVECHAMIENTO DEL FRUTO DE LA MELINA (Gmelina arborea ROXB) COMO COLORANTE NATURAL Y ANTIMICROBIANO}

\author{
Maximiliano Vanoye-Eligio ${ }^{1 / *}$, Leydi Leticia López-García ${ }^{2}$, José Alfredo García-Vela ${ }^{3}$, \\ José Alberto Alavez-Góngora ${ }^{4}$
}

Palabras clave: Melina; antimicrobiano; colorante; Campeche.

Keywords: Melina; antimicrobial; coloring; Campeche.

Recibido: 03/05/19

Aceptado: 24/07/19

RESUMEN

La melina es una especie forestal de origen asiático que se caracteriza por tener rápido crecimiento. Los usos más comunes que se le dan son para la elaboración de muebles, construcción estructural, obra liviana, carpintería y ebanistería, sin embargo, actualmente a los frutos de melina no se le ha dado un uso en la industria. Por ello, el objetivo de este trabajo fue el aprovechamiento integral de la melina para uso en la industria alimentaria como agente antimicrobiano y como un colorante natural para la industria textil. Esto se logró a través de un diseño experimental para la evaluación antimicrobiana y su uso como colorante. Los resultados indicaron que no existe diferencia significativa $(p<0,05)$ entre los tratamientos para determinar la capacidad antimicrobiana. En relación con el uso en la industria textilera, se detectó que el mordiente vinagre en los diferentes estados de

\footnotetext{
* Autor para correspondencia. Correo electrónico: maxvanoye@itsescarcega.edu.mx

1 Instituto Tecnológico Superior de Escárcega, Campeche, México. (iD) 0000-0002-6438-9479.

2 Instituto Tecnológico Superior de Escárcega, Campeche, México.
}

ABSTRACT
Use of the melina fruit (Gmelina
arboreal Roxb) as an antimicrobial and
natural colorant. The melina is a forest
species of Asian origin, is characterized by
rapid growth. The most common uses that are
given to this species are for the production of
furniture, structural construction, light work,
carpentry and joinery, however, currently the
melina fruit has not been used in industry,
therefore, the objective of this work was the
integral use of the melina for use in the food
industry as an antimicrobial agent, and its use
as a natural colorant for the textile industry.
This was achieved through an experimental
design for antimicrobial evaluation and its use
as a dye. The results indicated that there was
not significant difference (p<0.05) between
treatments to determine antimicrobial capacity.
In relation to its use in the textile industry,
ID $0000-0002-8999-8051$.
Instituto Tecnológico Superior de Escárcega,
Campeche, México.
(D) $0000-0001-9414-2154$.
anpeche, México.


madurez del fruto y en la corteza presenta una mayor coloración y uniformidad al teñir las telas de manta en donde existieron mejores resultados. Por ello, es importante considerar a la melina como una especie con fines maderables y no maderables. it was detected that vinegar mordant in the different states of ripeness of the fruit and in the rind had a greater coloration and uniformity when dyeing the fabrics, being the blanket fabric where better results existed. Therefore, it is important to consider the melina as a species with timber and non-timber purposes.

en el sureste mexicano el uso solo se limita a la extracción de madera y muy escasamente como forraje. Entre estos, el uso de las distintas partes de la especie (raíz, corteza, hojas, flores y frutos), la cuales presentan propiedades medicinales (Agrosoft 2000, Kawamura et al. 2005, Muñoz y Vera 2012, Azuero y Useche 2013).

Los estudios que se relacionan con la melina se encuentran principalmente encaminados al área forestal, sin embargo, es importante destacar que el aprovechamiento integral de la especie no se ha dado en México, por lo que es importante considerarla como potencial para la extracción de aceites esenciales que funjan como agentes antimicrobianos en la conservación de frutas y hortalizas de la región, ya que sus antecedentes taxonómicos la favorecen para la obtención de dichos agentes. De igual manera, la especie presenta un potencial para la obtención de un colorante natural a partir de los frutos, ya que integrantes de la población han mencionado que los frutos de la melina suelen manchar y que es muy difícil eliminar las manchas en el lugar afectado.

Hoy día existe la necesidad de buscar alternativas de conservación, debido al alto consumo de conservadores químicos en los alimentos que pueden afectar la salud, motivo que ha incrementado la demanda de productos frescos mínimamente tratados, lo que ha aumentado el interés por los conservadores de origen natural, tal como los agentes antimicrobianos (Sauceda 
2011). Por otro lado, entre los grandes problemas está el gran uso de colorantes sintéticos en la industria textil, que en su mayoría luego de ser utilizados sus desechos son depositados en cuerpos de aguas, contribuyendo a la contaminación de los mantos acuíferos y el medio ambiente y de igual forma, los colorantes usados en esta industria son difícilmente degradables y presentan oposición a tratamientos biológicos a que son sometidas las correspondientes aguas residuales (Gómez et al. 2011, Lin et al. 2011, Liu et al. 2011, Arévalo 2012). En los cuerpos de agua, los contaminantes de mayor impacto negativo son los colorantes sintéticos (Arango y Garcés 2009). Por lo que Martínez et al. (2009) promueve el uso de colorantes de origen natural, en especial del reino vegetal, ya que las más usadas son las provenientes de este, por lo que se puede trabajar con cortezas, hojas, raíces, frutos, cáscaras, semillas y flores. En el caso de las hojas, su mayor poder tintóreo lo presentan en la época de floración de la planta. Cuando se recolectan con anticipación, se conservan desecadas para su posterior utilización. En este caso los tonos obtenidos son más pálidos y opacos. Con respecto a los frutos, es ideal utilizarlos en su madurez y recolectar preferentemente aquellos que estén ya caídos.

\section{MATERIALES Y MÉTODOS}

Descripción del área de estudio: $\mathrm{El}$ trabajo de investigación se desarrolló en el municipio de Escárcega, Campeche. Geográficamente se encuentra ubicado entre los paralelos $18^{\circ} 37^{\prime} 00^{\prime \prime}$ de latitud norte y $90^{\circ} 43^{\prime} 00^{\prime \prime}$ de longitud oeste, a una altitud de 60 m (Cedeño 1981). El clima, de acuerdo con la clasificación de Köppen modificada por García (1988), es A1(i')g. en septiembre, y en octubre el municipio recibe la influencia de ciclones, y de noviembre a febrero se registran precipitaciones debidas a los nortes. La precipitación anual alcanza los $1390 \mathrm{~mm}$, la temperatura media anual es de $24,1^{\circ} \mathrm{C}$, con máximas y mínimas promedio de 32,1 y $15,9^{\circ} \mathrm{C}$, respectivamente (Centeno 1989). La vegetación existente en la zona de estudio es selva media y baja subperennifolia (Espinosa 1987).

Material vegetal: El material se recolectó en las plantaciones aledañas al municipio de Escárcega, tal es el caso de las plantaciones de Gmelina arborea que existen en la comunidad de Chekubul y Chicbul del municipio del Carmen, que se encuentran entre los 35 a 45 kilómetros aproximadamente. Las plantaciones son propiedad de la empresa Tka-blanca ubicada en la ciudad de Escárcega, que se dedica a la fabricación de chapas. De igual forma, se obtuvieron muestras en árboles que se encuentran en las instalaciones del Instituto Tecnológico Superior de Escárcega (ITSE) y del Colegio de Bachilleres del estado de Campeche (COBACAM) plantel 03 Escárcega; ambas ubicadas en la ciudad de Escárcega, Campeche. La colecta de frutos en sus distintos estados de madurez y el tronco se realizó con apoyo de la Bióloga Blanca del Rosario Martín Canché, quien ha trabajado con especies forestales en el estado de Campeche, México. Es importante destacar que la especie fue seleccionada debido a que es una de las principales especies comerciables en el estado de Campeche como producto maderable, sin embargo, también puede ser aprovechada en la categoría de productos no maderables.

Obtención de los extractos: En el laboratorio de Ingeniería en Industrias Alimentarias del Instituto Tecnológico Superior de Escárcega, las frutas se separaron de acuerdo con su estado de madurez (verde, intermedio y maduro), para de esta forma, obtener 3 diferentes extractos. $\mathrm{La}$ obtención de los extractos de la especie en estudio en sus diferentes estados de madurez consistió de varios pasos. Primeramente, se separó la pulpa de la semilla, ya que solo se trabajó con la pulpa, esto se realizó al partir el fruto a la mitad y extraer solo la pulpa; seguidamente, se licuó durante 5 minutos 200 gramos de pulpa con 300 mililitros de alcohol etílico. La muestra obtenida de la licuadora se filtró con una tela convencional, posteriormente, se colocó en un embudo de 
separación durante 24 horas con la finalidad de eliminar el mayor sedimento posible. Una vez que transcurrió el tiempo estimado, la muestra fue colocada en tubos de ensayo, los cuales tenían el mismo peso y se centrifugó a $35 \mathrm{rpm}$. durante $25 \mathrm{~min}$. Finalmente, dicha muestra fue colocada en el equipo de destilación por arrastre de vapor (rotovapor) hasta obtener el extracto de melina a un $95 \%$.

Evaluación de la actividad antimicrobiana: La capacidad antimicrobiana del extracto obtenido de la melina en sus distintos estados de madurez por arrastre de vapor, fue evaluada directamente en tomates a diferentes concentraciones, que fueron diluidas con agua (1:10, 1:50 y 1:100), además del testigo, al cual no se le agregó nada. Se incluyeron 3 repeticiones por cada concentración y el testigo. Cada repetición consistió en colocar 5 tomates en charola de unicel número 5 con $2,20 \mathrm{cms}$. de profundidad. Posteriormente, fueron rociadas con un atomizador moderada y uniformemente en cada repetición hechas a la intemperie. Es importante mencionar que los tomates fueron obtenidos de un centro comercial de la ciudad, que se encontraban en las mejores condiciones físicas. La evaluación de la capacidad antimicrobiana se realizó de manera visual cada 24 horas durante 5 días (96 horas). Los resultados fueron registrados en Excel versión 2010 y a través de este programa se realizó un análisis de varianza de una vía para comparar los diferentes tratamientos.

De igual manera, se realizaron ensayos microbiológicos con la finalidad de evaluar su capacidad antimicrobiana. Los sustratos utilizados fueron miel diluida (10\%), medio PDA y medio MacColkey como control positivo, es decir, esta facilitó el crecimiento de microorganismos. Las diluciones que se utilizaron de cada extracto de melina para las distintas etapas de madurez fueron $3(1 / 10,1 / 50$ y $1 / 100)$ y en cada sustrato se agregaba un $20 \%$ de la dilución, para un total de 27 repeticiones.
Evaluación de los extractos obtenidos para su aplicación como colorantes de origen natural en la industria textil: Se hiceron ensayos en diferentes estados de madurez del fruto de la melina (Gmelina arborea) para obtener el colorante. Primeramente se seleccionaron los frutos en los diferentes estados de madurez: verde, intermedio y maduro, de la misma manera se utilizaron hojas y la cáscara del tronco. En cada estado de madurez solo se trabajó con la pulpa, por lo que en cada tratamiento se usaron 300 gramos de pulpa, $300 \mathrm{~mL}$ de agua y 30 gramos del mordiente $(10 \%)$, obteniendo 6 tratamientos. Aunado a estos, también se trabajó con la corteza del árbol y las hojas con las mismas cantidades, lo cual sumó un total de 10 tratamientos. Para identificar fijación y tonalidades del colorante, se realizaron pruebas con algunos agentes químicos llamados mordientes, los cuales son de uso doméstico como sal y vinagre. Las proporciones de fruto, corteza y hojas con el agua fue de 1:1 y con un $10 \%$ del mordiente. Una vez obtenido el colorante en los diferentes estados de madurez y partes de la planta con los distintos mordientes, se introdujeron telas de algodón, manta y tela sintética en cada uno de los tratamientos obtenidos con el objeto de determinar la relación estado de madurez-mordiente-tela. Las telas introducidas en cada tratamiento se dejaron en reposo por una hora, posteriormente, estas se lavaron con jabón de ropa tradicional y se dejaron en secado para observar la fijación, coloración y tonalidad de cada tratamiento.

\section{RESULTADOS Y DISCUSIÓN}

Evaluación de la actividad antimicrobiana: Se trabajó con los frutos de la melina, corteza y hojas para valorar la capacidad antimicrobiana y su uso como colorante natural. Los frutos de la melina presentaron un diámetro de 1,9 a $3,7 \mathrm{~cm}$. En el caso de la valoración antimicrobiana de la especie a sus diferentes concentraciones (1:10, 1:50 y 1:100) se observó que no hay diferencia significativa de acuerdo al análisis estadístico (ANOVA) entre las 
concentraciones y el testigo, ya que el valor calculado de Fisher es menor que el valor crítico de Fisher (Valor crítico para F: 2.07335116-Valor F: 1.99325513), sin embargo, de manera cualitativa (visual) se pudo detectar que en el testigo, EI1:100 y EM1:100 hubo un deterioro en la calidad de la fruta a las 96 horas.

Sin embargo, en estudios anteriores, la Ruda (Ruta graveolens, R. chalepensis) ha demostrado inhibir el crecimiento de Alternaria solani Sorauer, hongo fitopatógeno que afecta principalmente a solanáceas y entre ellas al tomate (Solanum lycopersicum L.) y la papa (Solanum tuberosum L.) (Duarte et al. 2013). De igual manera, algunas especies de la familia Lamiaceae han demostrado capacidad antimicrobiana. Por su parte, Anesini y Perez (1993) estudiaron la actividad antibacteriana en extractos acuosos, en el cual obtuvieron resultados negativos y mostraron la actividad antimicrobiana del extracto en etanol al 40\% de las hojas del Psidium guajava L.

Los ensayos microbiológicos que se aplicaron a diferentes concentraciones y estados de madurez del extracto de melina para determinar su capacidad antimicrobiana en muestras positivas con cepas proliferadas para hongos y coliformes totales, se realizaron al considerar 3 repeticiones. Los resultados indican que las concentraciones 1:50 y 1:100 fueron las únicas que mostraron contaminación, es decir, no presentaron cualidades antimicrobianas, mientras que las otras concentraciones de los estados de madurez verde e intermedio sí mostraron cualidades antimicrobianas; sin embargo, estadísticamente no existe diferencia significativa entre los tratamientos. Así que en el área de industria alimentaria, específicamente en la conservación a la intemperie de frutas y verduras, no puede considerarse para la conservación de frutas y hortalizas que se comercialicen a la interperie.

Valoración de los extractos obtenidos para su aplicación como colorantes de origen natural en la industria textil: Los resultados indican que el fruto de la melina en un estado de madurez verde con mordiente vinagre ofrece un color beige más fuerte en comparación con los otros tratamientos, seguido por el tratamiento en estado de madurez intermedio con mordiente sal. Por otro lado, la uniformidad del color se observa mejor al utilizar el mordiente vinagre en comparación con la sal. Finalmente, los resultados de coloración y tonalidad se reflejaron mejor en la tela de manta, seguida por el algodón. En el caso de telas sintéticas, estas no se tiñeron con ningún tratamiento (Tabla 1). Por su parte, Pino et al. (2003) menciona que la sal fue el mordiente, que al mezclarse con los diferentes extractos vegetales, mostró mayor viscosidad en la tintura y fijación en las fibras y telas empleadas, seguido de la piedra de alumbre y en último lugar el vinagre. Como se puede observar, los resultados son contrastantes con los obtenidos por Pino et al. (2003). Por último, es importante destacar que el uso del colorante a partir de la melina solo fue utilizado para la industria textil, sin embargo, es necesario realizar pruebas en la industria de los alimentos, ya que se considera como un colorante potencial para su uso en dicha industria. 
Tabla 1. Resultado de la coloración y tonalidad obtenidas por los tratamientos de los estados de madurez del fruto y corteza de la melina.

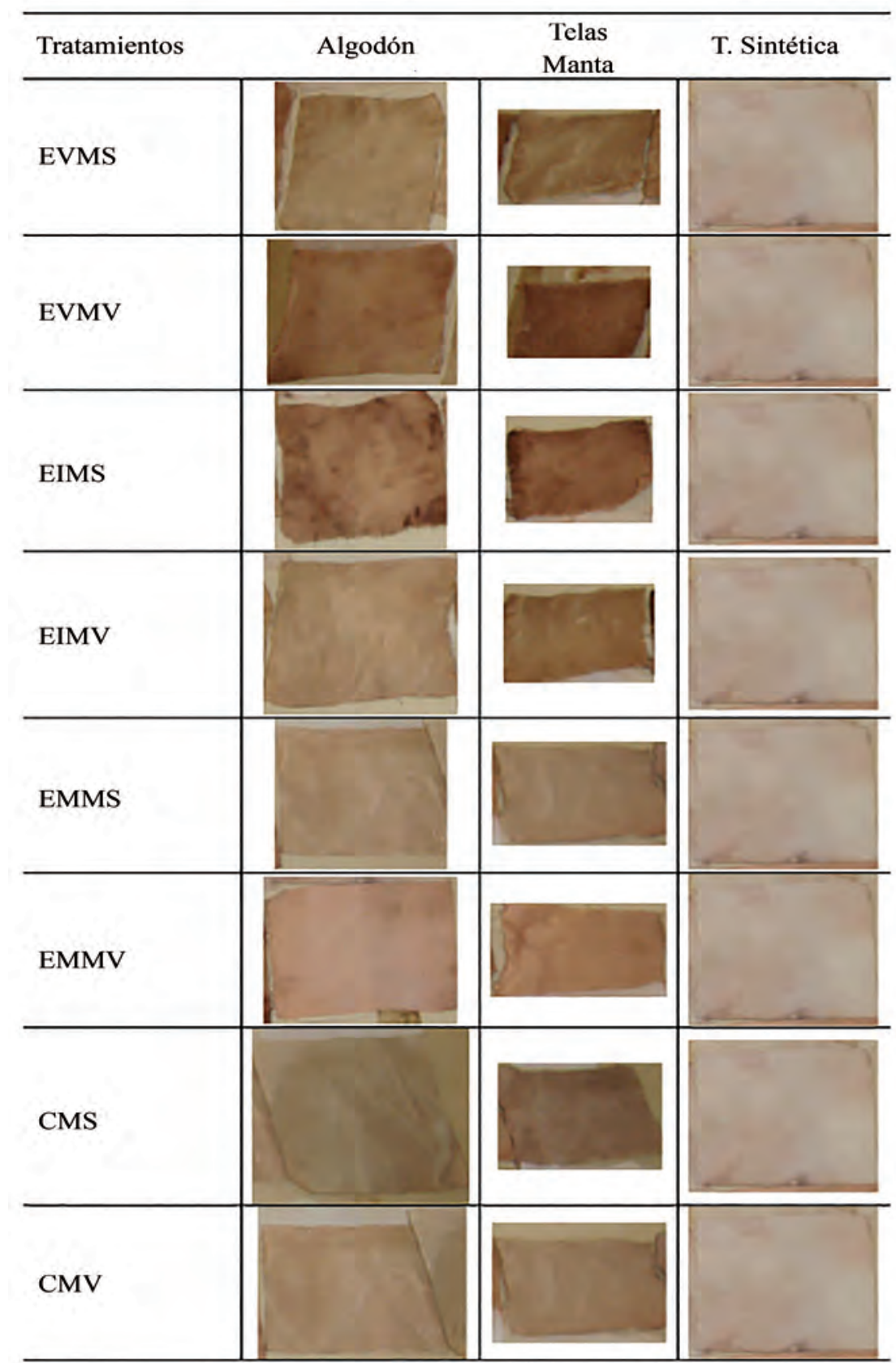

EVMS: Estado de madurez verde con mordiente sal; EVMV: Estado de madurez verde con mordiente vinagre; EIMS: Estado de madurez intermedio con mordiente sal; EIMV: Estado de madurez intermedio con mordiente vinagre; EMMS: Estado de madurez maduro con mordiente sal; EMMV: Estado de madurez maduro con mordiente vinagre; CMS: Corteza con mordiente sal; CMV: Corteza con mordiente vinagre. 


\section{CONCLUSIONES}

De acuerdo con el objetivo planteado en este trabajo de investigación, se pudo demostrar el potencial que tienen las plantaciones de melina en la industria textil como colorante, sin embargo, en la industria alimentaria no se considera una especie con capacidad antimicrobiana. Es importante mencionar que es necesario realizar pruebas para su uso como colorante en la industria alimentaria.

\section{AGRADECIMIENTOS}

Agradecimiento al Proyecto "Capacidad Antimicrobiana de Aceites Esenciales y/o extractos vegetales de especies aromáticas silvestres en el almacenamiento de frutas y verduras en Escárcega, Campeche, el cual fue aprobado ante TecNM con clave 260.15-PD en octubre de 2015. Asimismo, al Instituto Tecnológico Superior de Escárcega por brindar su apoyo en la ejecución del proyecto. Y de igual forma, las personas autoras agradecen al 2do Verano de la Ciencia Campeche, Selva, Mar, Cultura Maya y Ciencia organizado por COESICYDET - CONACYT.

\section{LITERATURA CITADA}

Agrosoft. 2000. Trees Versión 2. Árboles tropicales y subtropicales de uso múltiple. Reporte de especie $\mathrm{N}^{\mathrm{o}} .4$ Medellín, Colombia. $16 \mathrm{p}$.

Anesini, C; Perez, C. 1993. Screening of plants used in Argentine folk medicine for antimicrobial activity. Journal of Ethnopharmacology 39(2):119-128.

Arango, RA; Garcés, GLF. 2009. Remoción del colorante azoico amaranto de soluciones acuosas mediante electrocoagulación. Revista Lasallista de investigación 6(2):31-38.

Arévalo, CJM. 2012. Propuesta de un extracto colorante a partir de Hibiscus sabdariffa (Flor de Jamaica) para ser utilizada en la industria textil. Tesis Doctoral. Universidad de El Salvador. 113 p.

Azuero, RSA; Useche, TF. 2013. Yopo (Anadenanthera peregrina), Acacia (Acacia mangium Wild) y Melina (Melina arborea) tres especies arbóreas propicias para los sistemas silvopastoriles en el piedemonte llanero. Tesis Lic. Colombia, Universidad Nacional Abierta y a Distancia. 63 p.
Balcorta, MHC; Vargas, HJJ. 2004. Variación fenotípica y selección de árboles en una plantación de melina (Gmelina arborea Linn., Roxb.) de tres años de edad. Revista Chapingo. Serie Ciencias Forestales y del Ambiente 10(1):13-19.

Cedeño, SO. 1981. Campo Experimental Forestal "El Tormento", Campeche. Ciencia Forestal 1(3):75-82.

Centeno, ELR. 1989. Análisis estructural de cuatro etapas sucesionales de selva mediana subperennifolia en la región de Escárcega, Campeche. Tesis Lic. Chapingo, México, Universidad Autónoma Chapingo. $178 \mathrm{p}$.

Duarte, Y; Pino, O; Infante, D; Sánchez, Y; Travieso, MDC; Martínez, B. 2013. Efecto in vitro de aceites esenciales sobre Alternaria solani Sorauer. Revista de Protección Vegetal 28(1):54-59.

Enríquez, QM. 1978. Plantaciones con Gmelina arborea en el sureste de México para la producción de celulosa. In Memoria de la Primera Reunión Nacional de Plantaciones Forestales. SARH 13(1):110-117.

Espinosa, BA. 1987. Dinámica sinecológica de cuatro etapas sucesionales de una selva mediana subperennifolia en Escárcega, Campeche. Tesis Lic. Monterrey, N. L. México, Universidad Autónoma de Nuevo León. 115 p.

García, E. 1988. Modificaciones a la clasificación climática de Köppen (para adaptarlo a las condiciones de la República Mexicana). Instituto de Geografía. Universidad Nacional Autónoma de México, México. 252 p.

Gómez, ORT; Escudero, AMM; Gamarra, HZ. 2011. Tratamiento de efluentes textiles con luz ultravioleta solar. Industrial Data 14(2):9-15.

Kawamura, F; Ohara, S; Nishida, A. 2005. Antifungal activity of constituents from the heartwood of Gmelina arborea: Part 1. Sensitive antifungal assay against Basidiomycetes. Holzforschung 58(2):189-192.

Lin, Y; He, X; Han, G; Tian, Q; Hu. W. 2011. Removal of Crystal Violet from aqueous solution using powdered mycelial biomass of Ceriporia lacerata P2. Journal of Environmental Sciences 23(12):20552062.

Liu, X; Qiu, M; Huang, C. 2011. Degradation of the Reactive Black 5 by Fenton and Fenton-like system. Procedia Engineering 15:4835-4840.

Martínez, L; Álvarez, H; Del Val, S. 2009. Teñido de seda con colorantes naturales. Argentina: INTI. 26 p.

Muñoz, RML; Vera, RWA. 2012. Efectos de tres métodos pregerminativos y tres sustratos en la propagación de melina (Gmelina arborea roxb.) en el recinto Sabanetillas, cantón Echeandía, provincia Bolívar. Tesis Lic. Guaranda, Ecuador, Universidad Estatal de Bolívar. 177 p.

Pino Chalá, W; Guerrero, JE; Castro Rivas, A; Castro, AA; Palacios, JA; Castro, A. 2003. Extracción artesanal de colorantes naturales, una alternativa 
de aprovechamiento de la diversidad biológica del chocó, Colombia. Acta Biológica Colombiana 8(2):95-98.

Rodríguez, FR; Aguilar, DA; Roque, RM; Montoya, AM; Gamboa, OM; Arguedas, M. 2004. Manual para productores de melina Gmelina arborea en Costa Rica. Cartago, Costa Rica, Centro de Investigación en Integración Bosque Industria de la Escuela de Ingeniería Forestal del Instituto Tecnológico de Costa Rica. 314 p.

Roque, RM. 2004. Wood of Gmelina arborea in Costa Rica. New Forests 28(2-3):299-307.

Sampayo, MS; Silva, SMM; García, RJC. 2011. Establecimiento de plantaciones comerciales de melina (Gmelina arborea Roxb) en Tamaulipas. Desplegable para Productores. Instituto Nacional de Investigaciones Forestales, Agrícolas y Pecuarias. Centro de Investigación Regional del Noreste. Campo Experimental Río Bravo, México. $N^{0} 18$ Disponible en http://www.inifapcirne.gob.mx/ Biblioteca/Publicaciones/893.pdf

Sauceda, ENR. 2011. Uso de agentes antimicrobianos naturales en la conservación de frutas y hortalizas. Ra Ximhai 7(1):153-170.

Sosa, REE; Pérez, RD; Ortega, R; Zapata, BG. 2004. Evaluación del potencial forrajero de árboles y arbustos tropicales para la alimentación de ovinos. Técnica Pecuaria en México 42(2):129-144. 